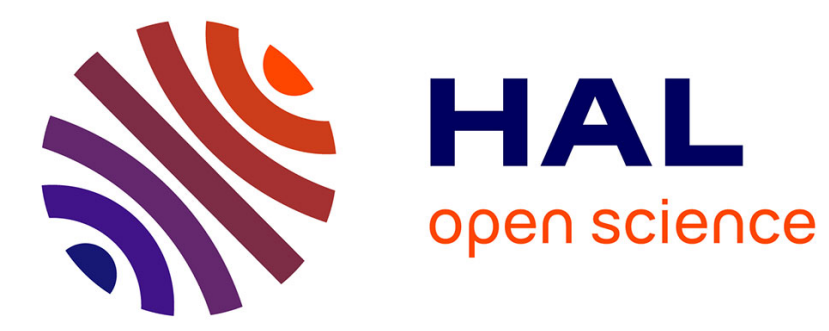

\title{
Nonautonomous fractional Hamiltonian system with critical exponential growth
}

João Marcos, Jacques Giacomoni, Pawan Kumar Mishra

\section{To cite this version:}

João Marcos, Jacques Giacomoni, Pawan Kumar Mishra. Nonautonomous fractional Hamiltonian system with critical exponential growth. Nonlinear Differential Equations and Applications, In press, 10.1007/s00030-019-0575-5 . hal-02160391

\section{HAL Id: hal-02160391 \\ https://hal.science/hal-02160391}

Submitted on 19 Jun 2019

HAL is a multi-disciplinary open access archive for the deposit and dissemination of scientific research documents, whether they are published or not. The documents may come from teaching and research institutions in France or abroad, or from public or private research centers.
L'archive ouverte pluridisciplinaire HAL, est destinée au dépôt et à la diffusion de documents scientifiques de niveau recherche, publiés ou non, émanant des établissements d'enseignement et de recherche français ou étrangers, des laboratoires publics ou privés. 


\title{
Nonautonomous fractional Hamiltonian system with critical exponential growth
}

\author{
João Marcos do Ó, Jacques Giacomoni and Pawan Kumar \\ Mishra
}

Abstract. In this paper, we study the following nonlocal nonautonomous Hamiltonian system on whole $\mathbb{R}$

$$
\begin{cases}(-\Delta)^{\frac{1}{2}} u+u=Q(x) g(v) & \text { in } \mathbb{R} \\ (-\Delta)^{\frac{1}{2}} v+v=P(x) f(u) & \text { in } \mathbb{R}\end{cases}
$$

where $(-\Delta)^{\frac{1}{2}}$ is the square root Laplacian operator. We assume that the nonlinearities $f, g$ have critical growth at $+\infty$ in the sense of TrudingerMoser inequality and the nonnegative weights $P(x)$ and $Q(x)$ vanish at $+\infty$. Using suitable variational method combined with the generalized linking theorem, we obtain the existence of at least one positive solution for the above system.

Mathematics Subject Classification (2010). Primary 35J50, 35R11, 35A15.

Keywords. elliptic systems involving square root of the Laplacian, critical growth nonlinearities of Trudinger-Moser type, linking theorem.

\section{Introduction and main results}

In this paper, we study the following system

$$
\begin{cases}(-\Delta)^{\frac{1}{2}} u+u=Q(x) g(v) & \text { in } \mathbb{R} \\ (-\Delta)^{\frac{1}{2}} v+v=P(x) f(u) & \text { in } \mathbb{R},\end{cases}
$$

where $(-\Delta)^{\frac{1}{2}}$ is the square root Laplacian operator defined as

$$
(-\Delta)^{\frac{1}{2}} u(x)=-\frac{1}{2 \pi} \int_{-1}^{1} \frac{u(x+y)+u(x-y)-2 u(x)}{|y|^{2}} d y .
$$

One difficulty in studying Hamiltonian elliptic systems via variational methods is that the energy functional is strongly indefinite, that is, its quadratic part is respectively coercive and anti-coercive in infinite dimensional subspaces of the energy space. To deal with such difficulty we use a Galerkin 
method, introduced by Rabinowitz in [25, 26]. Another important obstacle is to handle the lack of compactness, which roughly speaking, originates from the non-compactness of the Trudinger-Moser embedding. To add on, we face a lack of compactness of Sobolev embedding because the problem is posed in whole $\mathbb{R}$.

In the local case i.e. in the case of the standard Laplacian operator, the existence of solution for Hamiltonian elliptic systems has been extensively studied in the literature mostly in higher dimensions involving Sobolev critical growth in bounded as well as unbounded domain of $\mathbb{R}^{N}$. For the case of a bounded domain, see for instance, $[3,6,8,10,18,20]$. On the other hand, Hamiltonian elliptic systems in whole $\mathbb{R}^{N}$ have been explored to a lesser extend, see for example, $[11,22,29,30]$. We refer the reader to [4] for a recent survey on this subject.

For $N=2$, Hamiltonian systems in a bounded domain in $\mathbb{R}^{2}$ have been studied by D. G. de Figueiredo, J. M. do Ó and B. Ruf [7] in the critical growth range. We cite N. Lam and G. Lu [21] for a similar result without Ambrosetti-Rabinowitz condition (see $(H 3)$ ). In whole $\mathbb{R}^{2}$, D.G. de Figueiredo, J. M. do Ó and J. Zhang [9] studied the ground state solution for the system (1.1) with $P=Q=1$ using the idea of generalized Nehari manifold under a monotonicity assumption on nonlinearity i.e. $f(t) /|t|$ and $g(t) /|t|$ are strictly increasing in $(-\infty, 0)$ and $(0,+\infty)$. Another reference in unbounded domain is of M. de Souza and J. M. do Ó [13], where authors have considered a system of the type

$$
\begin{cases}-\Delta u+V(x) u=g(v) & \text { in } \mathbb{R}^{2}, \\ -\Delta v+V(x) v=f(u) & \text { in } \mathbb{R}^{2} .\end{cases}
$$

Under some suitable conditions on $V$, the loss of compactness in critical case was recovered.

We know from classical fractional Sobolev embedding that $H^{s, 2}\left(\mathbb{R}^{N}\right)$ is continuously embedded in $L^{q}\left(\mathbb{R}^{N}\right)$ for all $q \in\left[2,2_{s}^{*}\right]$, where $2_{s}^{*}=2 N /(N-2 s)$. Note that formally, $2_{s}^{*}=\infty$ if $N=2 s$. The only choice for this fact to be true is $N=1$ and $s=1 / 2$, since $s \in(0,1)$. At this point a natural question arises: What is the optimal space where $H^{1 / 2,2}(\mathbb{R})$ is embedded? This answer was first given by Ozawa [24] and later improved by Iula, Maalaoui and Martinazzi [23] which we have stated in Theorem A below.

In case of $u=v, f=g$ and $P=Q$, the system (1.1) converts into the following scalar equation

$$
(-\Delta)^{\frac{1}{2}} u+u=P(x) f(u) \text { in } \mathbb{R} .
$$

Motivated from fractional Trudinger Moser inequality as in Theorem A, J. M. do Ó, Miyagaki and Squassina [15] studied the existence of postive solutions to (1.2) with a class of weights $P$ containing the Lebesgue integrable functions and nonlinearities $f$ having subcritical and critical exponential growth (see also [16]). In the case of an open and bounded interval we cite an earlier work of Iannizzotto and Squassina [19], where authors have 
proved existence and multiplicity of positive solutions with critical exponential growth (see [17] also). We cite $[1,2,5,32,33]$ for more results in the case of Sobolev critical growth for dimension $N \geq 2$ involving the square root of Laplacian.

Inspired from the above literature, we consider in the present paper an Hamiltonian system involving the square root Laplacian operator posed in whole space $\mathbb{R}$ and investigate the effect of critical exponential growth of nonlinearities. We use a generalized linking theorem as compared to other results in the literature related to Hamiltonian systems which are heavily dependent on finite dimension Galerkin approximation to prove existence of at least one positive solution. For that, facing of the lack of compactness due to the critical growth of nonlinearities and unboundedness of the domain, we need to investigate accurately the behavior of suitable Palais Smale sequences. As far as we know there is no result regarding such class of fractional Hamiltonian systems. Our results appear to be the first of its kind for fractional Hamiltonian systems and we expect that the insights and the methods used in the present paper apply to a wider class of semilinear elliptic operators.

\subsection{Critical exponential nonlinearity}

It is usual in the literature to say that $h$ has critical growth of TrudingerMoser type at $+\infty$ if there exists $\alpha_{0}>0$ such that

$$
\lim _{s \rightarrow+\infty} \frac{h(s)}{e^{\alpha s^{2}}-1}=\left\{\begin{array}{ccc}
0 & \text { if } & \alpha>\alpha_{0}, \\
+\infty & \text { if } & \alpha<\alpha_{0} .
\end{array}\right.
$$

We note that such notion is motivated by a fractional version of TrudingerMoser inequality in the whole space $\mathbb{R}$ as follows.

Theorem A. (A fractional Trudinger-Moser inequality) It holds

$$
\sup _{u \in H^{1 / 2,2}(\mathbb{R}),\|u\|_{1 / 2} \leq 1} \int_{\mathbb{R}}\left(e^{\alpha|u|^{2}}-1\right) d x \begin{cases}<\infty, & \alpha \leq \pi, \\ =\infty, & \alpha>\pi,\end{cases}
$$

where $H^{1 / 2,2}(\mathbb{R})$ is the fractional order Sobolev space equipped with $\|\cdot\|_{1 / 2}$ norm which is defined in Section 2 .

\subsection{Assumptions on the weights $P(x)$ and $Q(x)$}

We assume $P, Q \not \equiv 0$ belonging to $C_{0}\left(\mathbb{R}, \mathbb{R}^{+}\right)$that is, $P, Q$ are continuous, nonnegative and

$$
\lim _{|x| \rightarrow+\infty} P(x)=0=\lim _{|x| \rightarrow+\infty} Q(x) .
$$

Without loss of generality, for the ease of reference, we assume:

$$
\sqrt{P(0) Q(0)}=1
$$

Remark 1.1. We recall that the assumption (1.3) does not imply that $P(x)$ and $Q(x)$ are Lebesgue integrable. For example take $P(x)=1$ if $|x| \leq 1$ otherwise $1 /|x|$. 
4 Joao Marcos do Ó, Jacques Giacomoni and Pawan Kumar Mishra

\subsection{Assumptions on the nonlinearities $f$ and $g$}

We consider the following assumptions on nonlinearities $f$ and $g$ which are $\alpha_{0}-$ critical at $+\infty$.

$(H 1)$ (continuity) $f, g: \mathbb{R} \rightarrow[0, \infty)$ are continuous functions and $f(0)=$ $g(0)=0$.

(H2) (behavior near the origin) $f(t)=o(t)$ and $g(t)=o(t)$ near the origin.

(H3) (Ambrosetti-Rabinowitz type condition) there exist constants $\theta>2$ such that, for all $t>0$, one has

$$
0<\theta F(t):=\theta \int_{0}^{t} f(s) \mathrm{d} s \leq t f(t) \text { and } 0<\theta G(t):=\theta \int_{0}^{t} g(s) \mathrm{d} s \leq t g(t) .
$$

(H4) (asymptotic behavior) $\lim _{t \rightarrow+\infty} t f(t) e^{-\alpha_{0} t^{2}}=+\infty$ and $\lim _{t \rightarrow+\infty} \operatorname{tg}(t) e^{-\alpha_{0} t^{2}}=+\infty$.

\subsection{Main result}

Before stating the main result of the paper, we define the following class of functions. We say that a function $f$ belongs to the class $\mathbb{C}_{\mathrm{P}}$, if for any sequence $\left\{u_{n}\right\} \subset H^{1 / 2,2}(\mathbb{R})$ satisfying for some positive constant $C$

$$
u_{n} \rightarrow 0 \text { and } \int_{\mathbb{R}} P(x) f\left(u_{n}\right) u_{n} \mathrm{~d} x<C \text { implies } \int_{\mathbb{R}} P(x) F\left(u_{n}\right) \mathrm{d} x \rightarrow 0 .
$$

Theorem 1.1. Assume $f$ and $g$ belong to the class $\mathbb{C}_{\mathrm{P}}$ and $\mathbb{C}_{\mathrm{Q}}$ respectively and have $\alpha_{0}$-critical growth at $+\infty$. Let $f$ and $g$ satisfy $(H 1)-(H 4)$ and $P$, $Q$ satisfy (1.3) then the system (1.1) possesses a positive solution.

Remark 1.2. The assumption (H5) given below together with (H2) implies that $f \in \mathbb{C}_{\mathrm{P}}$ and $g \in \mathbb{C}_{\mathrm{Q}}$.

(H5) $\lim _{t \rightarrow \infty} \frac{F(t)}{t f(t)}=0$ and $\lim _{t \rightarrow \infty} \frac{G(t)}{t g(t)}=0$.

We highlight that when $f$ and $g$ are of $O\left(t^{2}\right)$ near origin, $\mathbb{C}_{\mathrm{P}}$ implies the assumption (L6) adopted in [21, Theorem 4] in the critical case. However if $f$ and $g$ decay slowly at 0 , say are of $O\left(t^{1+\ell}\right)$ with $\ell<1, u_{n} \rightarrow 0$ and $\left\{\int_{\mathbb{R}} P(x) f\left(u_{n}\right) u_{n} d x\right\}$ is bounded do not imply that $\int_{\mathbb{R}} P(x) f\left(u_{n}\right) d x \rightarrow 0$. Precisely, vanishing behavior may occur for the sequence $\left\{P(x) f\left(u_{n}\right)\right\}$ in this case (see Remark 5.1). To validate our remark, we have added a proof in the appendix of this paper.

Remark 1.3. The result of Theorem 1.1 holds good even for the class of positive weights satisfying $P \in L^{\gamma}(\mathbb{R})$ and $Q \in L^{\gamma^{\prime}}(\mathbb{R})$ with $\gamma$, and $\gamma^{\prime}>1$ or $P, Q \in L^{1}(\mathbb{R}) \cap C(\mathbb{R}, \mathbb{R})$.

Remark 1.4. We have the following observations regarding the assumptions above.

(i) The assumption (H3) is a global Ambrosetti-Rabinowtiz condition which is used to prove the boundedness of the Palais-Smale sequence. 
(ii) Instead of (H4) one can take even a slightly weaker assumption $(H 4)^{\prime}$ : $\lim _{t \rightarrow+\infty} t f(t) e^{-\alpha_{0} t^{2}} \geq \eta$ and $\lim _{t \rightarrow+\infty} t g(t) e^{-\alpha_{0} t^{2}} \geq \eta$ where $\eta$ is a sufficiently large positive real number.

(iii) Examples of functions satisfying $(H 1)-(H 5)$ are $f(t)=t^{\ell_{1}} e^{t^{\beta_{1}}} e^{\alpha_{0} t^{2}}$, $g(t)=t^{\ell_{2}} e^{t^{\beta_{2}}} e^{\alpha_{0} t^{2}}$ with $\ell_{1}, \ell_{2}>1$ and $0 \leq \beta_{1}, \beta_{2}<2$.

Remark 1.5. We can consider different critical growth for $f$ and $g$, say $\alpha_{0}-$ and $\beta_{0}-$ critical growth respectively. Precisely, we can use the scaling $\bar{v}=$ $\sqrt{\frac{\beta_{0}}{\alpha_{0}}} v$ in (1.1) to get

$$
\begin{cases}(-\Delta)^{\frac{1}{2}} u+u=Q(x) \bar{g}(\bar{v}) & \text { in } \mathbb{R}, \\ (-\Delta)^{\frac{1}{2}} \bar{v}+\bar{v}=\bar{P}(x) f(u) & \text { in } \mathbb{R},\end{cases}
$$

where $\bar{P}(x)=\sqrt{\frac{\beta_{0}}{\alpha_{0}}} P(x)$ and $\bar{g}(\bar{v})=g\left(\sqrt{\frac{\alpha_{0}}{\beta_{0}}} v\right)$. It is easy to see that $\bar{g}$ has the $\alpha_{0}$ - critical growth as $g$ and $\bar{P} \in C_{0}(\mathbb{R}, \mathbb{R})$.

The paper is organized as follows. In section 2, we discuss the abstract framework related to the problem (1.1). A generalized version of Linking geometry and related estimates are shown in section 3. In particular, in Proposition 3.3, we give an upper estimate of the energy level that guarantees the compactness of suitable Palais Smale sequences. Here the assumption $(H 4)$ plays a crucial role. This estimate is subsequently used in Section 4 where the behavior of Palais Smale sequences is described. Finally, Section 5 contains the proof of the main result of Theorem 1.1.

\section{Abstract framework}

In order to apply variational methods, we recall the Bessel potential space $H^{s, p}\left(\mathbb{R}^{N}\right)$ as

$$
H^{s, p}\left(\mathbb{R}^{N}\right)=\left\{u \in L^{p}\left(\mathbb{R}^{N}\right):(-\Delta)^{s / 2} u \in L^{p}\left(\mathbb{R}^{N}\right)\right\} .
$$

On the other hand, the Sobolev-Slobodeckij space $W^{s, p}\left(\mathbb{R}^{N}\right)$ is defined as

$$
W^{s, p}\left(\mathbb{R}^{N}\right)=\left\{u \in L^{p}\left(\mathbb{R}^{N}\right):[u]_{s, p, \mathbb{R}^{N}}<\infty\right\},
$$

where

$$
[u]_{s, p, \mathbb{R}^{N}}^{p}=\int_{\mathbb{R}^{N}} \int_{\mathbb{R}^{N}} \frac{|u(x)-u(y)|^{p}}{|x-y|^{N+s p}} \mathrm{~d} x \mathrm{~d} y .
$$

In general, $H^{s, p}\left(\mathbb{R}^{N}\right) \neq W^{s, p}\left(\mathbb{R}^{N}\right)$ for $p \neq 2$. In this paper, we are interested in the limiting Sobolev embedding case i.e. when $2_{s}^{*}=2 N /(N-2 s)=\infty$ which, as observed before, corresponds to $s=1 / 2$ and $p=2$ in dimension $N=1$. In this case, the space $H^{1 / 2,2}(\mathbb{R})$ coincides with the SobolevSlobodeckij space $W^{1 / 2,2}(\mathbb{R})$ and both seminorms are related as

$$
\left\|(-\Delta)^{1 / 4} u\right\|_{L^{2}(\mathbb{R})}^{2}=\frac{1}{2 \pi}[u]_{W^{1 / 2,2}(\mathbb{R})}^{2},
$$


see Proposition 3.6. in [14]. The space $H^{1 / 2,2}(\mathbb{R})$ is the Hilbert space with the norm defined as

$$
\|u\|_{1 / 2}^{2}=\|u\|_{L^{2}(\mathbb{R})}^{2}+\int_{\mathbb{R}}\left|(-\Delta)^{\frac{1}{4}} u\right|^{2} \mathrm{~d} x
$$

induced from the inner product given as

$$
\langle u, v\rangle_{1 / 2}=\int_{\mathbb{R}}(-\Delta)^{\frac{1}{4}} u(-\Delta)^{\frac{1}{4}} v \mathrm{~d} x+\int_{\mathbb{R}} u v \mathrm{~d} x .
$$

We recall that $H^{1 / 2,2}(\mathbb{R})$ is continuously embedded in $L^{q}(\mathbb{R})$, for any $q \in$ $[2, \infty)$.

Consider the following weighted Banach space

$$
L^{r}(\mathbb{R} ; P)=\left\{u: \mathbb{R} \rightarrow \mathbb{R} \text { measurable: } \int_{\mathbb{R}} P(x)|u|^{r} \mathrm{~d} x<\infty\right\}
$$

and similarly we define

$$
L^{r}(\mathbb{R} ; Q)=\left\{u: \mathbb{R} \rightarrow \mathbb{R} \text { measurable: } \int_{\mathbb{R}} Q(x)|u|^{r} \mathrm{~d} x<\infty\right\} .
$$

We state our first result related to the compactness of the embedding of $H^{1 / 2,2}(\mathbb{R})$ into weighted integrable spaces defined above as follows.

Lemma 2.1. The space $H^{1 / 2,2}(\mathbb{R})$ is compactly embedded in $L^{q}(\mathbb{R} ; P)$ and $L^{q}(\mathbb{R} ; Q)$ respectively for $q \in[2, \infty)$.

Proof. We prove only the compact embedding of $H^{1 / 2,2}(\mathbb{R})$ into $L^{q}(\mathbb{R} ; P)$. The other one is similar. Let $q \in[2, \infty)$ and $\epsilon>0$. Then from the assumption (1.3), there exists $L=L(\epsilon)>0$ large enough, such that

$$
P(x)<\epsilon \text {, for all }|x|>L \text {. }
$$

If $\left\{u_{n}\right\} \subset H^{1 / 2,2}(\mathbb{R})$ is such that $u_{n} \rightarrow u$ weakly in $H^{1 / 2,2}(\mathbb{R})$ for some $u \in$ $H^{1 / 2,2}(\mathbb{R})$, then using the continuous injection of $H^{1 / 2,2}(\mathbb{R})$ in an arbitrary $L^{r}(\mathbb{R})$ space with $r \in[2, \infty)$ there exist $M, M_{r}>0$ such that

$$
\int_{\mathbb{R}}\left|(-\Delta)^{\frac{1}{4}} u_{n}\right|^{2} \mathrm{~d} x+\int_{\mathbb{R}}\left|u_{n}\right|^{2} \mathrm{~d} x \leq M, \int_{\mathbb{R}}\left|u_{n}\right|^{r} \mathrm{~d} x \leq M_{r} .
$$

Now from (2.1) and (2.2), we get

$$
\begin{aligned}
\int_{\mathbb{R} \backslash[-L, L]} P(x)\left|u_{n}\right|^{q} \mathrm{~d} x & \leq \epsilon \int_{\mathbb{R} \backslash[-L, L]}\left|u_{n}\right|^{q} \mathrm{~d} x \\
& \leq \epsilon \int_{\mathbb{R}}\left|u_{n}\right|^{q} \mathrm{~d} x \leq \epsilon\left\|u_{n}\right\|_{1 / 2}^{q} \leq \epsilon M^{q / 2} .
\end{aligned}
$$

Using in addition using the compact injection of $H^{1 / 2,2}(\mathbb{R})$ in an arbitrary $L_{\text {loc }}^{r}(\mathbb{R})$ space with $r \geq 2$ together with the boundedness of $P(x)$ in the compact subset of $\mathbb{R}$ as $P(x)$ is continuous, we have compactness of the embedding of $H^{1 / 2,2}(\mathbb{R})$ in $L^{q}(\mathbb{R} ; P)$ for all $q \in[2, \infty)$. 
Next, associated with our system we consider the Hilbert space $\mathbb{H}=$ $H^{1 / 2,2}(\mathbb{R}) \times H^{1 / 2,2}(\mathbb{R})$ with the inner product and norm

$$
\langle(u, v),(\varphi, \psi)\rangle:=\langle u, \varphi\rangle_{1 / 2}+\langle v, \psi\rangle_{1 / 2},\|(u, v)\|:=\left(\|u\|_{1 / 2}^{2}+\|v\|_{1 / 2}^{2}\right)^{1 / 2} .
$$

Consider the natural associated functional with system (1.1), defined in $\mathbb{H}$ by

$$
I(u, v):=\Phi(u, v)-\int_{\mathbb{R}} P(x) F(u) \mathrm{d} x-\int_{\mathbb{R}} Q(x) G(v) \mathrm{d} x,
$$

where the associated quadratic part is defined by

$$
\Phi(u, v):=\int_{\mathbb{R}}\left((-\Delta)^{\frac{1}{4}} u(-\Delta)^{\frac{1}{4}} v+u v\right) \mathrm{d} x .
$$

Using standard arguments it is possible to verify that $I$ is well defined and is of class $C^{1}$ with

$$
\begin{gathered}
I^{\prime}(u, v)(\phi, \psi)=\int_{\mathbb{R}}\left((-\Delta)^{\frac{1}{4}} u(-\Delta)^{\frac{1}{4}} \psi+(-\Delta)^{\frac{1}{4}} v(-\Delta)^{\frac{1}{4}} \phi+u \psi+v \phi\right) \mathrm{d} x \\
-\int_{\mathbb{R}}(P(x) f(u) \phi+Q(x) g(v) \psi) \mathrm{d} x \quad \forall(\phi, \psi) \in \mathbb{H} .
\end{gathered}
$$

Consequently, critical points of the functional $I$ are precisely the weak solutions to (1.1).

Note that the functional $I$ is strongly indefinite, since $\Phi(u, v)>0$ when $0 \not \equiv(u, v) \in \mathbb{H}^{+}$and $\Phi(u, v)<0$ whenever $0 \not \equiv(u, v) \in \mathbb{H}^{-}$, where $\mathbb{H}^{+}:=\left\{(u, u): u \in H^{1 / 2,2}(\mathbb{R})\right\}, \mathbb{H}^{-}:=\left\{(u,-u): u \in H^{1 / 2,2}(\mathbb{R})\right\}$ are infinite dimensional subspaces of $\mathbb{H}$.

In order to deal with the fact that the functional $I$ is strongly indefinite, we shall use a version of the Palais-Smale condition inspired by the Galerkin method which we describe next.

\subsection{Palais-Smale condition and Generalized Mountain Pass Theorem}

Let $\mathbb{W}$ be a real separable Banach space, and suppose $\left\{\phi_{i}: i \in J \subset \mathbb{N}\right\}$ is a basis of $\mathbb{W}$. Given a family $\left\{J_{n}\right\}_{n \in \mathbb{N}}$ such that $J_{1} \subset J_{2} \subset \cdots J_{n} \subset \cdots J$, and $\cup_{n \in \mathbb{N}} J_{n}=J$, we set for every $n \in \mathbb{N}$,

$$
\mathbb{W}_{n}=\overline{\operatorname{span}\left\{\phi_{i}: i \in J_{n}\right\}} \quad \text { and } \quad I_{n}=\left.I\right|_{\mathbb{W}_{n}}
$$

Now we shall consider the $(P S)^{*}$ condition with respect to the family $\left(\mathbb{W}_{n}\right)$ of $\mathbb{W}$ in the following sense:

Definition 2.1. Given $c \in \mathbb{R}$, we say that $\left(u_{n}\right) \subset \mathbb{W}$ is a $(P S)_{c}^{*}$ sequence for the functional $I \in C^{1}(\mathbb{W}, \mathbb{R})$ if

(i). There exists a sequence $\left(n_{j}\right) \subset \mathbb{N}, n_{j} \rightarrow \infty$ as $j \rightarrow \infty$ such that $u_{n_{j}} \in \mathbb{W}_{n_{j}}$, for every $j \in \mathbb{N}$;

(ii). $I\left(u_{n_{j}}\right) \rightarrow$ c as $j \rightarrow \infty$;

(iii). $\left\|I_{n_{j}}^{\prime}\left(u_{n_{j}}\right)\right\|_{\mathbb{W}_{n_{j}}^{*}} \rightarrow 0$, as $j \rightarrow \infty$.

Definition 2.2. Given $c \in \mathbb{R}$, we say that the functional $I \in C^{1}(\mathbb{W}, \mathbb{R})$ satisfies the $(P S)_{c}^{*}$ condition (with respect to the family $\left(\mathbb{W}_{n}\right)$ ) if every $(P S)_{c}^{*}$ sequence for the functional I possesses a subsequence converging to a critical point of $I$. 
We apply the following version of Generalized Mountain Pass Theorem ( see [27] and [28]). Considering $\mathbb{W}=X_{1} \oplus X_{2}$, we shall suppose $I \in C^{1}(\mathbb{W}, \mathbb{R})$ satisfies the $(P S)_{c}^{*}$ condition with respect to the family $\left(\mathbb{W}_{n}\right) \subset \mathbb{W}$ with $\mathbb{W}_{n}=X_{1}^{n} \oplus X_{2}^{n}, X_{i}^{n} \subset X_{i}, i=1,2, \operatorname{dim}\left(X_{1}^{n}\right)<\infty$ for any $n \in \mathbb{N}$.

Theorem 2.2. (Generalized Mountain Pass Theorem) Let $\mathbb{W}=X_{1} \oplus X_{2}$ be a real Banach space. Suppose $I \in C^{1}(\mathbb{W}, \mathbb{R})$ satisfies

$\left(I_{0}\right)$. For every $u \in X_{1}$, it holds $I(u) \leq 0$.

$\left(I_{1}\right)$. There exist $\rho>0$ and $\sigma>0$ such that

$$
I(u) \geq \sigma, \quad \text { for every } u \in \partial B_{\rho}(0) \cap X_{2} .
$$

$\left(I_{2}\right)$. For each $n \in \mathbb{N}$, there exist $e_{n} \in \partial B_{1}(0) \cap X_{2}^{n}$ and $\beta \in \mathbb{R}$ such that

$$
I(u) \leq \beta, \quad \text { for every } u \in X_{1} \oplus \mathbb{R}^{+} e_{n},
$$

where

$$
X_{1} \oplus \mathbb{R}^{+} e_{n}=\left\{u=v+t e_{n} \in X_{1} \oplus \mathbb{R} e_{n}: v \in X_{1}, t \geq 0\right\} .
$$

If I satisfies $(P S)_{c}^{*}$ for every $c \in[\sigma, \beta]$, then I possesses a critical point $u \in \mathbb{W}$ such that $I(u) \in[\sigma, \beta]$.

\section{The linking geometry and estimates for the critical level}

In this section we verify that the functional $I$, defined in (2.3), satisfies the geometrical properties of Theorem 2.2. We start by verifying the hypothesis $\left(I_{0}\right)$ and $\left(I_{1}\right)$.

Lemma 3.1. There exist $\rho, \sigma>0$ such that $I(z) \geq \sigma$, for all $z \in S:=$ $\partial B_{\rho} \cap \mathbb{H}^{+}$. Moreover, $I(z) \leq 0$ if $z \in \mathbb{H}^{-}$.

Proof. Assumption (H2) implies that, for a given $\epsilon_{0}>0$, there exists $t_{0}>0$ such that

$$
f(t) \leq 2 \epsilon_{0} t \text { and } F(t) \leq \epsilon_{0} t^{2} \text {, for all } t \leq t_{0} .
$$

On the other hand, given $\alpha>\alpha_{0}$ and $q>2$ there exists a positive constant $C_{1}$ such that,

$$
F(t) \leq C_{1} t^{q}\left(\exp \alpha t^{2}-1\right), \text { for all } t \geq t_{0} .
$$

Given $u \in H^{1}(\mathbb{R})$ and define $\Omega_{t_{0}}:=\left\{x \in \mathbb{R}: 0 \leq u(x) \leq t_{0}\right\}$. Thus,

$$
\begin{aligned}
\int_{\mathbb{R}} P(x) F(u) \mathrm{d} x & =\int_{\Omega_{t_{0}}} P(x) F(u) \mathrm{d} x+\int_{\mathbb{R} \backslash \Omega_{t_{0}}} P(x) F(u) \mathrm{d} x \\
& \leq \epsilon_{0} \int_{\mathbb{R}} P(x) u^{2} \mathrm{~d} x+C_{1} \int_{\mathbb{R} \backslash \Omega_{t_{0}}} P(x) u^{q}\left(\exp \alpha u^{2}-1\right) \mathrm{d} x .
\end{aligned}
$$

Now using the Hölder's inequality with $r^{-1}+s^{-1}=1$, we obtain

$$
\begin{aligned}
\int_{\mathbb{R}} P(x) F(u) \mathrm{d} x & \leq \epsilon_{0}\|u\|_{L^{2}(\mathbb{R} ; P)}^{2} \\
& +C_{1}\|u\|_{L^{q r}(\mathbb{R} ; P)}^{q}\left(\int_{\mathbb{R} \backslash \Omega_{t_{0}}} P(x)\left(\exp \alpha u^{2}-1\right)^{s} \mathrm{~d} x\right)^{\frac{1}{s}} .
\end{aligned}
$$


We use the following inequality to estimate the second term in (3.1). There exists a constant $C_{2}=C_{2}\left(t_{0}\right)$ such that

$$
\left(\exp \alpha t^{2}-1\right)^{s} \leq C_{2}\left(\exp \alpha s t^{2}-1\right) \text { for all } t \geq t_{0}
$$

which implies

$$
\int_{\mathbb{R} \backslash \Omega_{t_{0}}} P(x)\left(\exp \left(\alpha u^{2}\right)-1\right)^{s} \mathrm{~d} x \leq C_{2} \int_{\mathbb{R} \backslash \Omega_{t_{0}}} P(x)\left(\exp \left(\alpha s u^{2}\right)-1\right) \mathrm{d} x .
$$

From (1.3), we have

$$
\int_{\mathbb{R} \backslash \Omega_{t_{0}}} P(x)\left(\exp \alpha s u^{2}-1\right) \mathrm{d} x \leq C \int_{\mathbb{R}}\left(\exp \alpha s u^{2}-1\right) \mathrm{d} x
$$

which applied to (3.1) together with Lemma 2.1 gives

$$
\int_{\mathbb{R}} P(x) F(u) \mathrm{d} x \leq \epsilon_{0} C\|u\|_{1 / 2}^{2}+C_{3}\|u\|_{1 / 2}^{q}\left(\int_{\mathbb{R}}\left(\exp \alpha s u^{2}-1\right) \mathrm{d} x\right)^{\frac{1}{s}} .
$$

Analogously, possibly for different positive constants $C$ and $C_{3}$, we have

$$
\int_{\mathbb{R}} Q(x) G(u) \mathrm{d} x \leq \epsilon_{0} C\|u\|_{1 / 2}^{2}+C_{3}\|u\|_{1 / 2}^{q}\left(\int_{\mathbb{R}}\left(\exp \alpha s u^{2}-1\right) \mathrm{d} x\right)^{\frac{1}{s}} .
$$

The previous inequalities used in (2.3) imply

$$
I(u, u) \geq\|u\|_{1 / 2}^{2}-C_{1} \epsilon_{0}\|u\|_{1 / 2}^{2}-C_{4}\|u\|_{1 / 2}^{q}\left(\int_{\mathbb{R}}\left(\exp \alpha s u^{2}-1\right) \mathrm{d} x\right)^{\frac{1}{s}} .
$$

The Trudinger-Moser inequality as in Theorem A reads

$$
\int_{\mathbb{R}}\left(\exp \alpha s u^{2}-1\right) \mathrm{d} x=\int_{\mathbb{R}}\left\{\exp \left[\alpha s\|u\|_{1 / 2}^{2}\left(\frac{u}{\|u\|_{1 / 2}}\right)^{2}\right]-1\right\} \mathrm{d} x \leq C
$$

whenever $\|u\|_{1 / 2}^{2}=\rho$ and $\alpha s \rho<\pi$ (we can choose $\rho$ sufficiently small for this to hold good). Hence

$$
I(u, u) \geq\|u\|_{1 / 2}^{2}-C_{1} \epsilon_{0}\|u\|_{1 / 2}^{2}-C_{5}\|u\|_{1 / 2}^{q} .
$$

Therefore, since $q>2$, we can find $\sigma>0$ sufficiently small, such that $I(u, u) \geq \sigma>0$ for $\|u\|_{1 / 2}=\rho$. To complete the proof, one can see that for any $z=(u,-u) \in \mathbb{H}^{-}$,

$$
I(u,-u)=-\|u\|_{1 / 2}^{2}-\int_{\mathbb{R}}[P(x) F(u)+Q(x) G(u)] \mathrm{d} x \leq 0,
$$

by using assumption $(H 1)$.

Now we obtain an upper bound for the minimax level, and consequently $I$ satisfies $\left(I_{2}\right)$. The argument strongly relies on the Trudinger-Moser inequality and the growth condition $(H 4)$. This estimate depends on an intricate reasoning involving the Moser sequence to be introduced. The idea is similar 
to that found in the celebrated work due to H. Brezis and L. Nirenberg. However here we replace the Talenti's functions by the Moser's functions, defined as truncations and dilations of the fundamental solution: given $k \in \mathbb{N}$,

$$
m_{k}(x)=\frac{\bar{\omega}_{k}(x)}{\left\|\bar{\omega}_{k}\right\|_{1 / 2}},
$$

where

$$
\bar{\omega}_{k}(x)=\frac{1}{\sqrt{\pi}} \begin{cases}(\log k)^{1 / 2}, & |x| \leq 1 / k, \\ \frac{\log \frac{1}{|x|}}{(\log k)^{1 / 2}}, & 1 / k \leq|x| \leq 1, \\ 0, & |x| \geq 1 .\end{cases}
$$

Lemma 3.2. Defining $\rho_{k}:=\frac{\log k}{\pi}-m_{k}^{2}$, we have that there exists $C_{o}>0$ such that

$$
0 \leq \rho_{k} \leq C_{o} \quad \text { for every } \quad|x| \leq \frac{1}{k}
$$

Proof. Indeed, we have

$$
\left\|\bar{\omega}_{k}\right\|_{1 / 2}^{2}=\int_{\mathbb{R}}\left|(-\Delta)^{\frac{1}{4}} \bar{\omega}_{k}(x)\right|^{2} \mathrm{~d} x+\int_{B(0,1)}\left|\bar{\omega}_{k}(x)\right|^{2} \mathrm{~d} x=1+r_{k} .
$$

Here $r_{k} \geq 0$ and direct calculations yield $r_{k}=O\left(\frac{1}{\log k}\right)$ as $k \rightarrow \infty$ (see estimates $(2.3)-(2.5)$ in [31]). Hence, $\left|r_{k} \log k\right| \leq C$ for $k>k_{0}$ leading to $\lim _{k \rightarrow \infty} r_{k}=0$. Therefore, if $|x| \leq 1 / k$, from the definition of $m_{k}$ and (3.2), we get

$$
m_{k}^{2}:=\frac{\log k}{\pi\left\|\bar{\omega}_{k}\right\|_{1 / 2}^{2}}=\frac{\log k}{\pi\left(1+r_{k}\right)}=\frac{\log k}{\pi}-\frac{(\log k) r_{k}}{\pi\left(1+r_{k}\right)}
$$

Hence

$$
\rho_{k}=\frac{(\log k) r_{k}}{\pi\left(1+r_{k}\right)}
$$

which together with the logarithmic decay estimate on $r_{k}$, as above, completes the proof.

Proposition 3.3. There exists $l_{0} \in \mathbb{N}$ such that for all $l \geq l_{0}$ the corresponding Moser's function $m_{l}$ satisfies

$$
\sup _{\mathbb{R}^{+}\left(m_{l}, m_{l}\right) \oplus \mathbb{H}^{-}} I<\frac{\pi}{\alpha_{0}} .
$$

Proof. Suppose by contradiction that there exists a sequence $\left(l_{k}\right) \subset \mathbb{N}$ such that $l_{k} \nearrow+\infty$ and for every $k \in \mathbb{N}$,

$$
\sup _{\mathbb{R}^{+}\left(m_{l_{k}}, m_{l_{k}}\right) \oplus \mathbb{H}^{-}} I \geq \frac{\pi}{\alpha_{0}} .
$$

So, for every $k \in \mathbb{N}, j \in \mathbb{N}$, there exists $u_{j, k} \in H^{1 / 2,2}(\mathbb{R})$ and $\tau_{j, k}>0$ such that

$$
I\left(\eta_{j, k}\right) \geq \frac{\pi}{\alpha_{0}}-\frac{1}{j},
$$


where

$$
\eta_{j, k}=\tau_{j, k}\left(m_{l_{k}}, m_{l_{k}}\right)+\left(u_{j, k},-u_{j, k}\right) \in \mathbb{R}^{+}\left(m_{l_{k}}, m_{l_{k}}\right) \oplus \mathbb{H}^{-} .
$$

Let $h:[0, \infty) \rightarrow \mathbb{R}$ be defined by $h(t):=I\left(t \eta_{j, k}\right)$. Since $h(0)=0$ and $\lim _{t \rightarrow+\infty} h(t)=-\infty$, there exists a maximum point $t_{o} \in(0, \infty)$ such that

$$
h\left(t_{o}\right)=I\left(t_{o} \eta_{j, k}\right) \geq \frac{\pi}{\alpha_{0}}-\frac{1}{j} .
$$

Without loss of generality we may assume that $t_{o}=1$. Hence

$$
I^{\prime}\left(\eta_{j, k}\right) \eta_{j, k}=0
$$

Using (3.3) and (3.4), we can write

$$
\begin{aligned}
\tau_{j, k}^{2} \geq \frac{\pi}{\alpha_{0}}- & \frac{1}{j}+\left\|u_{j, k}\right\|_{1 / 2}^{2}+\int_{\mathbb{R}} P(x) F\left(\tau_{j, k} m_{l_{k}}+u_{j, k}\right) \mathrm{d} x \\
& +\int_{\mathbb{R}} Q(x) G\left(\tau_{j, k} m_{l_{k}}-u_{j, k}\right) \mathrm{d} x
\end{aligned}
$$

and

$$
\begin{aligned}
2 \tau_{j, k}^{2}= & 2\left\|u_{j, k}\right\|_{1 / 2}^{2}+\int_{\mathbb{R}} P(x) f\left(\tau_{j, k} m_{l_{k}}+u_{j, k}\right)\left(\tau_{j, k} m_{l_{k}}+u_{j, k}\right) \mathrm{d} x \\
& +\int_{\mathbb{R}} Q(x) g\left(\tau_{j, k} m_{l_{k}}-u_{j, k}\right)\left(\tau_{j, k} m_{l_{k}}-u_{j, k}\right) \mathrm{d} x
\end{aligned}
$$

From (3.5) it follows

$$
\frac{\pi}{\alpha_{0}}+s_{j, k}:=\tau_{j, k}^{2} \geq \frac{\pi}{\alpha_{0}}-\frac{1}{j} .
$$

Therefore $\liminf \operatorname{in}_{j \rightarrow \infty} s_{j, k} \geq 0$.

Consider $C_{o}>0$ given by Lemma 3.2 and take $\beta_{0}>0$ be such that

$$
\beta_{0}>\frac{\pi}{2 \alpha_{0}} \exp \left(\pi C_{o}\right)
$$

By $(H 4)$, there exists $R_{0}=R_{0}\left(\beta_{0}\right)>0$ such that

$$
t f(t) e^{-\alpha_{0} t^{2}} \geq \beta_{0} \text { and } \operatorname{tg}(t) e^{-\alpha_{0} t^{2}} \geq \beta_{0}, \quad \text { for all } t \geq R_{0} .
$$

Therefore there exists $C>0$ such that $\operatorname{tf}(t) \geq \beta_{0} e^{\alpha_{0} t^{2}}-C$ and $\operatorname{tg}(t) \geq$ $\beta_{0} e^{\alpha_{0} t^{2}}-C$ for $t \geq 0$. We also take $\bar{s}>0$ and $k_{1} \in \mathbb{N}$ such that for all $s \geq \bar{s}$ and $k \geq k_{1}$

$$
\begin{aligned}
& 2\left(\frac{\pi}{\alpha_{0}}+s\right) \exp \left(\pi C_{o}\right) \\
& <\beta_{0} l_{k} \exp \left(\alpha_{0} s\left(\frac{\log l_{k}}{\pi}-\rho_{l_{k}}\right)\right) \int_{B_{1 / l_{k}}(0)} \sqrt{P(x) Q(x)} \mathrm{d} x .
\end{aligned}
$$


12 Joao Marcos do Ó, Jacques Giacomoni and Pawan Kumar Mishra

Next we use the inequality $e^{x}+e^{y} \geq 2 e^{\frac{x+y}{2}}$ to get the following estimate

$$
\begin{aligned}
& \int_{B_{1 / l_{k}}(0)}\left(P(x) f\left(\tau_{j, k} m_{l_{k}}+u_{j, k}\right)+Q(x) g\left(\tau_{j, k} m_{l_{k}}-u_{j, k}\right)\right)\left(\tau_{j, k} m_{l_{k}}-u_{j, k}\right) \mathrm{d} x \\
& \geq \beta_{0} \int_{B_{1 / l_{k}}(0)} P(x) \exp \left(\alpha_{0}\left(\tau_{j, k} m_{l_{k}}+u_{j, k}\right)^{2}\right) \mathrm{d} x \\
& \quad+\beta_{0} \int_{B_{1 / l_{k}}(0)} Q(x) \exp \left(\alpha_{0}\left(\tau_{j, k} m_{l_{k}}-u_{j, k}\right)^{2}\right) \mathrm{d} x-O\left(1 / l_{k}\right) \\
& \geq 2 \beta_{0} \int_{B_{1 / l_{k}}(0)} \sqrt{P(x) Q(x)} \exp \left(\alpha_{0}\left(\tau_{j, k} m_{l_{k}}\right)^{2}\right) \mathrm{d} x-O\left(1 / l_{k}\right) .
\end{aligned}
$$

From (3.6), (3.7), (3.8), (3.10) and Lemma 3.2,

$$
\begin{aligned}
& \frac{\pi}{\alpha_{0}}+s_{j, k} \\
& =\tau_{j, k}^{2} \geq \beta_{0} \int_{B_{1 / l_{k}}(0)} \sqrt{P(x) Q(x)} \exp \left(\alpha_{0} \tau_{j, k}^{2}\left(\frac{\log l_{k}}{\pi}-\rho_{l_{k}}\right)\right)-O\left(1 / l_{k}\right) \\
& \quad \geq \beta_{0} l_{k} \exp \left(-\pi \rho_{l_{k}}\right) \exp \left(\alpha_{0} s_{j, k}\left(\frac{\log l_{k}}{\pi}-\rho_{l_{k}}\right)\right) \int_{B_{1 / l_{k}}(0)} \sqrt{P(x) Q(x)} \mathrm{d} x \\
& \quad-O\left(1 / l_{k}\right)
\end{aligned}
$$

which is equivalent to

$$
\begin{aligned}
& \left(\frac{\pi}{\alpha_{0}}+s_{j, k}\right) \exp \left(\pi \rho_{l_{k}}\right) \\
& \geq \beta_{0} l_{k} \exp \left(\alpha_{0} s_{j, k}\left(\frac{\log l_{k}}{\pi}-\rho_{l_{k}}\right)\right) \int_{B_{1 / l_{k}}(0)} \sqrt{P(x) Q(x)} \mathrm{d} x-O\left(1 / l_{k}\right) .
\end{aligned}
$$

Without loss of generality, we can assume that for $k \geq k_{1}$ one has $\log l_{k}>$ $2 C_{o} \pi$. Then we claim that for every $k \geq k_{1}$, we have $s_{j, k} \leq \bar{s}$. Indeed, otherwise from Lemma 3.2,

$$
\begin{aligned}
& 2\left(\frac{\pi}{\alpha_{0}}+s_{j, k}\right) \exp \left(\pi C_{o}\right) \\
& \geq \beta_{0} l_{k} \exp \left(\alpha_{0} s_{j, k}\left(\frac{\log l_{k}}{\pi}-\rho_{l_{k}}\right)\right) \int_{B_{1 / l_{k}}(0)} \sqrt{P(x) Q(x)} \mathrm{d} x \\
& \geq \beta_{0} l_{k} \exp \left(\alpha_{0} s_{j, k} C_{o}\right) \int_{B_{1 / l_{k}}(0)} \sqrt{P(x) Q(x)} \mathrm{d} x .
\end{aligned}
$$

However, this contradicts (3.9). The claim is proved.

In view of the above claim and (3.7), we have

$$
-\frac{1}{j} \leq s_{j, k} \leq \bar{s} \quad \text { for every } k \geq k_{1} .
$$

Thus, taking a subsequence if necessary, we may suppose that

$$
\lim _{j \rightarrow \infty} s_{j, k}=s_{o, k} \in[0, \bar{s}] \quad \text { for every } k \geq k_{1} .
$$


Consequently, from (3.11), for every $k \geq k_{1}$,

$$
\begin{gathered}
\left(\frac{\pi}{\alpha_{0}}+s_{o, k}\right) \exp \left(\pi \rho_{l_{k}}\right) \\
\geq \beta_{0} l_{k} \exp \left(\alpha_{0} s_{o, k}\left(\frac{\log l_{k}}{\pi}-\rho_{l_{k}}\right)\right) \int_{B_{1 / l_{k}}(0)} \sqrt{P(x) Q(x)} \mathrm{d} x-O\left(1 / l_{k}\right) .
\end{gathered}
$$

Hence, since $s_{o, k} \geq 0$ and from Lemma 3.2, we get

$$
\begin{gathered}
\left(\frac{\pi}{\alpha_{0}}+s_{o, k}\right) \exp \left(\pi C_{o}\right) \exp \left(\alpha_{0} s_{o, k} C_{o}\right) \\
\geq \beta_{0} l_{k} \exp \left(\alpha_{0} s_{o, k}\left(\frac{\log l_{k}}{\pi}\right)\right) \int_{B_{1 / l_{k}}(0)} \sqrt{P(x) Q(x)} \mathrm{d} x-O\left(1 / l_{k}\right) .
\end{gathered}
$$

Note that, from (3.12) and $s_{o, k} \in[0, \bar{s}]$ we have that $s_{o, k} \rightarrow 0$ as $k \rightarrow \infty$. Consequently, taking $k \rightarrow \infty$ in (3.12) and using (1.4), we get

$$
\frac{\pi}{2 \alpha_{0}} \exp \left(\pi C_{o}\right) \geq \beta_{0} \text {. }
$$

However, this contradicts our choice of $\beta_{0}$. Thus Proposition 3.3 is proved.

\section{The $(P S)_{c}^{*}$ condition}

In this section we shall verify that the functional $I$ satisfies the $(P S)_{c}^{*}$ condition with respect to an appropriate family of subspaces $\left(\mathbb{H}_{n}\right)_{n}$ of $\mathbb{H}$. As a consequence of compact embedding results as in Lemma 2.1, there exists an orthonormal basis $\left\{\varphi_{1}, \varphi_{2} \ldots\right\}$ in $H^{1 / 2,2}(\mathbb{R})$ of normalized eigenfunctions associated to the eigenvalues of the compact operator $\left((-\Delta)^{-1 / 2}, P\right)$ in the weighted $L^{2}(\mathbb{R} ; P)$ space. Set,

$$
\begin{aligned}
& \mathbb{E}_{n}^{+}=\operatorname{span}\left\{\left(\varphi_{i}, \varphi_{i}\right): i=1, \ldots, n\right\} \\
& \mathbb{E}_{n}^{-}=\operatorname{span}\left\{\left(\varphi_{i},-\varphi_{i}\right): i=1, \ldots, n\right\}
\end{aligned}
$$

Considering $e=m_{k}$ given by Proposition 3.3, we define

$$
\begin{aligned}
\mathbb{H}_{n} & =\mathbb{H}_{n}^{+} \oplus \mathbb{H}_{n}^{-} \quad \text { and } I_{n}=\left.I\right|_{\mathbb{H}_{n}}, \quad \text { where } \\
\mathbb{H}_{n}^{+} & =\mathbb{R}(e, e) \oplus \mathbb{E}_{n}^{+}, \\
\mathbb{H}_{n}^{-} & =\mathbb{R}(e,-e) \oplus \mathbb{E}_{n}^{-} .
\end{aligned}
$$

Remark 4.1. We observe that it is easy to see that if $z:=(u, v) \in \mathbb{H}_{n}$ then $(v, 0) \in \mathbb{H}_{n}$ and $(0, u) \in \mathbb{H}_{n}$.

Next result establishes that $(P S)^{*}$ sequences are bounded.

Proposition 4.1. Given $c \in \mathbb{R}$, let $\left\{z_{n}\right\}:=\left\{\left(u_{n}, v_{n}\right)\right\} \subset \mathbb{H}$ be a $(P S)_{c}^{*}$ sequence for the functional $I$ with respect to the family $\left(\mathbb{H}_{n}\right)_{n}$ defined above. Then $\left\{z_{n}\right\}$ is bounded in $\mathbb{H}$. Furthermore, there exists $C_{1}>0$ such that

$$
\begin{aligned}
\int_{\mathbb{R}} P(x) f\left(u_{n}\right) u_{n} \mathrm{~d} x & \leq C_{1}, & \int_{\mathbb{R}} Q(x) g\left(v_{n}\right) v_{n} \mathrm{~d} x \leq C_{1}, \\
\int_{\mathbb{R}} P(x) F\left(u_{n}\right) \mathrm{d} x & \leq C_{1}, & \int_{\mathbb{R}} Q(x) G\left(v_{n}\right) \mathrm{d} x \leq C_{1} .
\end{aligned}
$$


14 Joao Marcos do Ó, Jacques Giacomoni and Pawan Kumar Mishra

Proof. The sequence $\left\{z_{n}\right\} \subset \mathbb{H}$ being a $(P S)_{c}^{*}$ sequence for $I$ satisfies the following
(a) $I\left(u_{n}, v_{n}\right) \rightarrow c$ and
(b) $\left\|I_{n}^{\prime}\left(u_{n}, v_{n}\right)\right\|_{\mathbb{H}_{n}^{*}} \rightarrow 0$, as $n \rightarrow \infty$.

Taking $(\varphi, \psi)=\left(u_{n}, v_{n}\right) /\left\|\left(u_{n}, v_{n}\right)\right\| \in \mathbb{H}_{n}$ as testing functions according to (b), we have for some sequence $\left(\varepsilon_{n}\right)_{n \in \mathbb{N}}$ tending to 0 :

$$
\begin{gathered}
\left|2 \int_{\mathbb{R}}\left((-\Delta)^{\frac{1}{4}} u_{n}(-\Delta)^{\frac{1}{4}} v_{n}+u_{n} v_{n}\right) \mathrm{d} x-\int_{\mathbb{R}}\left(P(x) f\left(u_{n}\right) u_{n}+Q(x) g\left(v_{n}\right) v_{n}\right) \mathrm{d} x\right| \\
\leq \varepsilon_{n}\left\|\left(u_{n}, v_{n}\right)\right\|
\end{gathered}
$$

which together with (b) and (H3) imply for some sequence $\left(\delta_{n}\right)_{n \in \mathbb{N}}$ tending to 0 and $\theta>2$

$$
\begin{aligned}
& \int_{\mathbb{R}} P(x) f\left(u_{n}\right) u_{n} \mathrm{~d} x+\int_{\mathbb{R}} Q(x) g\left(v_{n}\right) v_{n} \mathrm{~d} x \\
& \leq 2 \int_{\mathbb{R}} P(x) F\left(u_{n}\right) \mathrm{d} x+\int_{\mathbb{R}} Q(x) G\left(v_{n}\right) \mathrm{d} x+2 c+2 \delta_{n}+\varepsilon_{n}\left\|\left(u_{n}, v_{n}\right)\right\| \\
& \leq \frac{2}{\theta} \int_{\mathbb{R}} P(x) f\left(u_{n}\right) u_{n} \mathrm{~d} x+\int_{\mathbb{R}} Q(x) g\left(v_{n}\right) v_{n} \mathrm{~d} x+2 c+2 \delta_{n}+\varepsilon_{n}\left\|\left(u_{n}, v_{n}\right)\right\| .
\end{aligned}
$$

Thus

$$
\int_{\mathbb{R}} P(x) f\left(u_{n}\right) u_{n} \mathrm{~d} x+\int_{\mathbb{R}} Q(x) g\left(v_{n}\right) v_{n} \mathrm{~d} x \leq \frac{\theta}{\theta-2}\left(1+2 \delta_{n}+\varepsilon_{n}\left\|\left(u_{n}, v_{n}\right)\right\|\right) .
$$

Next taking $(\varphi, \psi)=\left(v_{n}, 0\right) /\left\|v_{n}\right\|_{1 / 2}$ and $(\varphi, \psi)=\left(0, u_{n}\right) /\left\|u_{n}\right\|_{1 / 2}$ in (b) we have

$$
\begin{aligned}
\left\|v_{n}\right\|_{1 / 2}^{2}-\varepsilon_{n}\left\|v_{n}\right\|_{1 / 2} & \leq \int_{\mathbb{R}} P(x) f\left(u_{n}\right) v_{n} \mathrm{~d} x \\
\left\|u_{n}\right\|_{1 / 2}^{2}-\varepsilon_{n}\left\|u_{n}\right\|_{1 / 2} & \leq \int_{\mathbb{R}} Q(x) g\left(v_{n}\right) u_{n} \mathrm{~d} x .
\end{aligned}
$$

Setting $U_{n}=u_{n} /\left\|u_{n}\right\|_{1 / 2}$ and $V_{n}=v_{n} /\left\|v_{n}\right\|_{1 / 2}$ we have

$$
\begin{aligned}
\left\|v_{n}\right\|_{1 / 2} & \leq \int_{\mathbb{R}} P(x) f\left(u_{n}\right) V_{n} \mathrm{~d} x+\varepsilon_{n}, \\
\left\|u_{n}\right\|_{1 / 2} & \leq \int_{\mathbb{R}} Q(x) g\left(v_{n}\right) U_{n} \mathrm{~d} x+\varepsilon_{n} .
\end{aligned}
$$

We now rely on the following Young type inequality ( see [12], Lemma 2.4):

$$
s t \leq \begin{cases}\left(e^{t^{2}}-1\right)+s\left(\log ^{+} s\right)^{1 / 2}, & \text { for all } t \geq 0 \text { and } s \geq e^{1 / 4} \\ \left(e^{t^{2}}-1\right)+\frac{1}{2} s^{2}, & \text { for all } t \geq 0 \text { and } 0 \leq s \leq e^{1 / 4} .\end{cases}
$$

Using the critical growth of $f$ and $g$, for any $\epsilon>0$, there exists a constant $C_{1}=C_{1}(\epsilon)>0$ such that

$$
f(s) \leq C_{1} e^{\alpha_{0}(1+\epsilon) s^{2}} \quad \text { and } \quad g(s) \leq C_{1} e^{\left(\alpha_{0}(1+\epsilon) s^{2}\right.} \quad \text { for all } \quad s \in \mathbb{R} .
$$


Let $A_{n}=\left\{x \in \mathbb{R}: \frac{1}{C_{1}} f\left(u_{n}\right)(x) \geq e^{1 / 4}\right\}, B_{n}=\left\{x \in \mathbb{R}: \frac{1}{C_{1}} f\left(u_{n}\right)(x) \leq e^{1 / 4}\right\}$. Then

$$
\begin{aligned}
& \int_{\mathbb{R}} P(x) f\left(u_{n}\right) V_{n} \mathrm{~d} x \leq C_{1} \int_{\mathbb{R}} P(x)\left(e^{V_{n}^{2}}-1\right) \mathrm{d} x+ \\
& \int_{A_{n}} P(x) f\left(u_{n}\right)\left[\log \frac{1}{C_{1}} f\left(u_{n}\right)\right]^{1 / 2} \mathrm{~d} x+\frac{1}{2 C_{1}} \int_{B_{n}} P(x)\left(f\left(u_{n}\right)\right)^{2} \mathrm{~d} x .
\end{aligned}
$$

By (4.4), the second integral on the right hand side yields

$$
\begin{aligned}
\int_{A_{n}} P(x) f\left(u_{n}\right)\left[\log \frac{1}{C_{1}} f\left(u_{n}\right)\right]^{1 / 2} \mathrm{~d} x & \leq \int_{A_{n}} P(x) f\left(u_{n}\right)\left[\log e^{\alpha_{0}(1+\epsilon) u_{n}^{2}}\right]^{1 / 2} \mathrm{~d} x \\
& \leq \sqrt{\alpha_{0}(1+\epsilon)} \int_{\mathbb{R}} P(x) f\left(u_{n}\right) u_{n} \mathrm{~d} x .
\end{aligned}
$$

Note that by using condition $(H 2)$, for some fixed $s_{o}>0$ there exists a positive constant $C=C\left(s_{o}\right)$ we have $f(s) \leq C s$ for $0<s<s_{o}$. Therefore,

$$
f(s)^{2} \leq C f(s) s \text { for all } 0 \leq s \leq s_{o}
$$

and

$$
f(s)^{2} \leq \frac{C_{1} e^{1 / 4}}{s_{o}} f(s) s \quad \text { for all } \quad s \geq s_{o} \quad \text { when } \quad f(s) \leq C_{1} e^{1 / 4}
$$

which implies

$$
\begin{aligned}
\int_{B_{n}} P(x)\left[f\left(u_{n}\right)\right]^{2} \mathrm{~d} x= & \int_{\left\{x \in B_{n}: u_{n}(x) \in\left[0, s_{o}\right]\right\}} P(x)\left(f\left(u_{n}\right)\right)^{2} \mathrm{~d} x \\
& +\int_{\left\{x \in B_{n}: u_{n}(x) \geq s_{o}\right\}} P(x)\left(f\left(u_{n}\right)\right)^{2} \mathrm{~d} x \\
\leq & C \int_{\mathbb{R}} P(x) f\left(u_{n}\right) u_{n} \mathrm{~d} x .
\end{aligned}
$$

Now, in the light of Theorem A, the first integral in the right hand side of (4.5) is bounded i.e.

$$
C_{1} \int_{\mathbb{R}} P(x)\left(e^{V_{n}^{2}}-1\right) \mathrm{d} x \leq C .
$$

Substituting the above estimates in (4.5), we obtain for some positive constant $C$

$$
\int_{\mathbb{R}} P(x) f\left(u_{n}\right) V_{n} \mathrm{~d} x \leq C\left(1+\int_{\mathbb{R}} P(x) f\left(u_{n}\right) u_{n} \mathrm{~d} x\right) .
$$

This estimate together with (4.1)-(4.2) imply

$$
\left\|v_{n}\right\|_{1 / 2} \leq C\left(1+2 \delta_{n}+\varepsilon_{n} \cdot\left\|\left(u_{n}, v_{n}\right)\right\|\right) .
$$

Repeating the argument above it follows

$$
\left\|u_{n}\right\|_{1 / 2} \leq C\left(1++2 \delta_{n}+\varepsilon_{n} \cdot\left\|\left(u_{n}, v_{n}\right)\right\|\right) .
$$

Now considering the estimates (4.6) and (4.7) we finally obtain

$$
\left\|\left(u_{n}, v_{n}\right)\right\| \leq C
$$


which completes the proof.

Proposition 4.2. Let $c \in\left(0, \pi / \alpha_{0}\right)$ and $\left\{z_{n}\right\} \subset \mathbb{H}$ be a $(P S)_{c}^{*}$ sequence for the functional $I$, with respect to the family $\left(\mathbb{H}_{n}\right)_{n}$ of $\mathbb{H}$, then $\left\{z_{n}\right\}$ possesses a subsequence which converges weakly in $\mathbb{H}$ to a critical point of $I$.

Proof. By Proposition 4.1, $\left\{z_{n}\right\}$ is bounded sequence in $\mathbb{H}$. Hence, invoking Lemma 2.1 and the fact that $\mathbb{H}$ is a Hilbert space, $\left\{z_{n}\right\}$ has a subsequence (still denote by $\left\{z_{n}\right\}$ ) such that

$$
\begin{array}{rll}
z_{n} & := & \left(u_{n}, v_{n}\right) \rightarrow z_{o}:=\left(u_{o}, v_{o}\right) \text { in } \mathbb{H}, \\
u_{n} \rightarrow u_{o} & \text { and } & v_{n} \rightarrow v_{o} \text { in } L^{q}(\mathbb{R} ; P) \text { and } L^{q}(\mathbb{R} ; Q), \forall q \in[2, \infty), \\
u_{n}(x) \rightarrow u_{o}(x) \quad \text { and } & v_{n}(x) \rightarrow v_{o}(x) \text { a.e. in } \mathbb{R} .
\end{array}
$$

We also note that, in view of Proposition 4.1, there exists $C>0$ such that, for every $n \in \mathbb{N}$,

$$
\int_{\mathbb{R}} P(x) f\left(u_{n}\right) u_{n} \mathrm{~d} x \leq C, \quad \int_{\mathbb{R}} Q(x) g\left(v_{n}\right) v_{n} \mathrm{~d} x \leq C .
$$

We claim that for any $\phi \in C_{0}^{\infty}(\mathbb{R})$ with $\operatorname{supp} \phi=K \subset \subset \mathbb{R}$, we have

$$
\int_{\mathbb{R}} P(x) f\left(u_{n}\right) \phi \mathrm{d} x \rightarrow \int_{\mathbb{R}} P(x) f\left(u_{o}\right) \phi \mathrm{d} x .
$$

Indeed, let $M>0$ and $\psi_{M}$ a smooth cut-off function such that

$$
\psi_{M}(t)= \begin{cases}1 & \text { for }|t| \leq M \\ 0 & \text { for }|t| \geq M+1\end{cases}
$$

Then we have

$$
\begin{aligned}
& \int_{\mathbb{R}} P(x) f\left(u_{n}\right) \phi \mathrm{d} x \\
& =\int_{\mathbb{R}} P(x)\left(1-\psi_{M}\left(u_{n}\right)\right) f\left(u_{n}\right) \phi \mathrm{d} x+\int_{\mathbb{R}} P(x) \psi_{M}\left(u_{n}\right) f\left(u_{n}\right) \phi \mathrm{d} x \\
& =\int_{\mathbb{R} \cap\left\{u_{n}>M\right\}} P(x)\left(1-\psi_{M}\left(u_{n}\right)\right) f\left(u_{n}\right) \phi \mathrm{d} x \\
& \quad+\int_{\mathbb{R} \cap\left\{u_{n}<M+1\right\}} P(x) \psi_{M}\left(u_{n}\right) f\left(u_{n}\right) \phi \mathrm{d} x \\
& \leq \frac{\|\phi\|_{\infty}}{M} \int_{\mathbb{R}} P(x) f\left(u_{n}\right) u_{n} \mathrm{~d} x+\int_{\mathbb{R} \cap\left\{u_{n}<M+1\right\}} P(x) \psi_{M}\left(u_{n}\right) f\left(u_{n}\right) \phi \mathrm{d} x .
\end{aligned}
$$

In the above inequality, using the Lebesgue dominated convergence theorem in the second integral and (4.9) in the first integral, we get for large $n$

$$
\int_{\mathbb{R}} P(x) f\left(u_{n}\right) \phi \mathrm{d} x \leq \frac{C\|\phi\|_{\infty}}{M}+\int_{\mathbb{R} \cap\left\{u_{0} \leq M+1\right\}} P(x) \psi_{M}\left(u_{0}\right) f\left(u_{0}\right) \phi \mathrm{d} x .
$$


Now we estimate the second integral, keeping in mind the fact that $\psi_{M}\left(u_{n}\right) \rightarrow$ 1 a.e. in $\mathbb{R}$ for $M$ large enough as

$$
\begin{gathered}
\left|\int_{\mathbb{R}} P(x) f\left(u_{0}\right) \phi \mathrm{d} x-\int_{\mathbb{R}} P(x) \psi_{M}\left(u_{0}\right) f\left(u_{0}\right) \phi \mathrm{d} x\right| \\
\leq \frac{\|\phi\|_{\infty}}{M} \int_{\mathbb{R}} P(x) f\left(u_{0}\right) u_{0} \mathrm{~d} x=O(1 / M) .
\end{gathered}
$$

Now for large $M>0$, we combine (4.12) with (4.11) to get our required result. Similarly one can show that

$$
\int_{\mathbb{R}} Q(x) g\left(v_{n}\right) \psi \mathrm{d} x \rightarrow \int_{\mathbb{R}} Q(x) g\left(v_{o}\right) \psi \mathrm{d} x .
$$

Finally using (4.8), (4.10) and (4.13) we conclude that

$$
I^{\prime}\left(u_{o}, v_{o}\right)(\phi, \psi)=0 \text { for all }(\phi, \psi) \in C_{0}^{\infty}(\mathbb{R}) \times C_{0}^{\infty}(\mathbb{R}) .
$$

Using the fact that $\cup \mathbb{H}_{n}$ is dense in $\mathbb{H}$ it follows that (4.14) holds for any $(\phi, \psi) \in \mathbb{H}$. In other words, $z_{o}$ is a critical point of $I$. Proposition 4.2 is now proved.

\section{Proof of Theorem 1.1}

Arguing by contradiction, we suppose that the origin is the only critical point of the functional $I$. Note that the positivity of nontrivial weak solutions follows from the classical regularity theory and strong maximum principle for fractional Laplacian problems.

We shall verify that, under this assumption, $I$ satisfies $(P S)_{c}^{*}$ for every $c \in\left(0, \pi / \alpha_{0}\right)$.

Let $\left\{z_{n}\right\}=\left\{\left(u_{n}, v_{n}\right)\right\} \in \mathbb{H}_{n}$ be such that

$$
\begin{gathered}
I\left(z_{n}\right) \rightarrow c \in\left(0, \pi / \alpha_{0}\right), \\
\left\|I_{n}^{\prime}\left(z_{n}\right)\right\|_{\mathbb{H}_{n}^{*}} \rightarrow 0 .
\end{gathered}
$$

By Lemma 2.1, Propositions 3.3, 4.1 and the fact that the origin is the only possible critical point of $I$, we may assume that there exists $C>0$ such that

$$
\begin{aligned}
\left\|z_{n}\right\| & \leq C, \text { in } \mathbb{H} \\
z_{n}=\left(u_{n}, v_{n}\right) & \rightarrow(0,0) \text { weakly in } \mathbb{H}, \\
u_{n} & \rightarrow 0 \text { strongly in } L^{q}(\mathbb{R} ; P), \forall q \in[2, \infty), \\
v_{n} & \rightarrow 0 \text { strongly in } L^{q}(\mathbb{R} ; Q), \forall q \in[2, \infty), \\
z_{n}(x)=\left(u_{n}(x), v_{n}(x)\right) & \rightarrow(0,0) \text { a. e. in } \mathbb{R}, \\
\int_{\mathbb{R}} P(x) f\left(u_{n}\right) u_{n} \mathrm{~d} x \leq C, & \int_{\mathbb{R}} Q(x) g\left(v_{n}\right) v_{n} \mathrm{~d} x \leq C .
\end{aligned}
$$

Since $f$ and $g$ belong to the class $\mathbb{C}_{\mathrm{P}}$ and $\mathbb{C}_{\mathrm{Q}}$ respectively, we get

$$
\int_{\mathbb{R}} P(x) F\left(u_{n}\right) \mathrm{d} x \rightarrow 0, \quad \int_{\mathbb{R}} Q(x) G\left(v_{n}\right) \mathrm{d} x \rightarrow 0 .
$$


From (5.5) and (5.1), we may find $\delta>0$ such that, for $n$ sufficiently large,

$$
\int_{\mathbb{R}}\left((-\Delta)^{\frac{1}{4}} u_{n}(-\Delta)^{\frac{1}{4}} v_{n}+u_{n} v_{n}\right) \mathrm{d} x<\frac{\pi}{\alpha_{0}}-2 \delta .
$$

This inequality, combined with (5.2) and (5.3), implies, for $n$ sufficiently large,

$$
\int_{\mathbb{R}} P(x) f\left(u_{n}\right) u_{n} \mathrm{~d} x+\int_{\mathbb{R}} Q(x) g\left(v_{n}\right) v_{n} \mathrm{~d} x \leq \frac{\pi}{\alpha_{0}}-\delta .
$$

We claim that, given $\beta>\alpha_{0}$, for $n$ sufficiently large,

$$
\left\|u_{n}\right\|_{1 / 2}+\left\|v_{n}\right\|_{1 / 2} \leq\left(\frac{\beta}{\alpha_{0}}\left(\frac{\pi}{\alpha_{0}}-\delta\right)\right)^{1 / 2} .
$$

In order to prove this claim we note that $\left\|u_{n}\right\|_{1 / 2} \not \rightarrow 0$. Indeed, assuming otherwise, from (5.3) we get that

$$
\lim _{n \rightarrow \infty} \int_{\mathbb{R}}\left((-\Delta)^{\frac{1}{4}} u_{n}(-\Delta)^{\frac{1}{4}} v_{n}+u_{n} v_{n}\right) \mathrm{d} x=0
$$

which together with (5.1) and (5.5), leads to a contradiction. Thus, we assume that $\left\|u_{n}\right\|_{1 / 2} \geq b>0$ and $\left\|v_{n}\right\|_{1 / 2} \geq b>0$ for all $n$. Now, set $\tilde{u}_{n}=\left(\frac{\pi}{\alpha_{0}}-\right.$ $\delta)^{1 / 2} \frac{u_{n}}{\left\|u_{n}\right\|_{1 / 2}}, s=\frac{g\left(v_{n}\right)}{\sqrt{\alpha_{0}}}$ and $t=\sqrt{\alpha}_{0} \tilde{u}_{n}$. Then, using (5.2) and (4.3) with $s$ and $t$ as above results into

$$
\begin{aligned}
& \left(\frac{\pi}{\alpha_{0}}-\delta\right)^{1 / 2}\left\|u_{n}\right\|_{1 / 2} \\
& =\int_{\mathbb{R}} Q(x) g\left(v_{n}\right) \tilde{u}_{n} \mathrm{~d} x+o_{n}(1) \leq \int_{\mathbb{R}} Q(x)\left(e^{\alpha_{0} \tilde{u}_{n}^{2}}-1\right) \mathrm{d} x \\
& \quad+\int_{\left\{x \in \mathbb{R}: g\left(v_{n}\right)(x) \geq \sqrt{\alpha_{0}} e^{1 / 4}\right\}} Q(x) \frac{g\left(v_{n}\right)}{\sqrt{\alpha_{0}}}\left[\log \left(\frac{g\left(v_{n}\right)}{\sqrt{\alpha_{0}}}\right)\right]^{1 / 2} \mathrm{~d} x \\
& \quad+\frac{1}{2} \int_{\left\{x \in \mathbb{R}: g\left(v_{n}\right)(x) \leq \sqrt{\alpha_{0}} e^{1 / 4}\right\}} Q(x) \frac{\left(g\left(v_{n}\right)\right)^{2}}{\alpha_{0}} \mathrm{~d} x+o_{n}(1) .
\end{aligned}
$$

Let us evaluate each integral on the right hand side of (5.8). Taking $p>1$ and using the elementary inequalities for real numbers

$$
\begin{aligned}
e^{\alpha_{0} t^{2}}-1-\alpha_{0} t^{2} & \leq \alpha_{0} t^{2}\left(e^{\alpha_{0} t^{2}}-1\right) \\
\left(e^{\alpha_{0} t^{2}}-1\right)^{p} & \leq C\left(e^{p \alpha_{0} t^{2}}-1\right), \quad \text { for } t \geq 0,
\end{aligned}
$$

together with the Hölder inequality, we obtain

$$
\begin{aligned}
& \int_{\mathbb{R}} Q(x)\left(e^{\alpha_{0} \tilde{u}_{n}^{2}}-1-\alpha_{0}{\tilde{u_{n}}}^{2}\right) \mathrm{d} x \\
& \leq \alpha_{0}\left\|\tilde{u}_{n}\right\|_{L^{2 s}(\mathbb{R} ; Q)}^{2}\left[\int_{\mathbb{R}} Q(x)\left(e^{\alpha_{0} \tilde{u}_{n}^{2}}-1\right) s^{s^{\prime}} \mathrm{d} x\right]^{1 / s^{\prime}} \\
& \leq C \alpha_{0}\left\|\tilde{u}_{n}\right\|_{L^{2 s}(\mathbb{R} ; Q)}^{2}\left[\int_{\mathbb{R}}\left(e^{\alpha_{0} s^{\prime} \tilde{u}_{n}^{2}}-1\right) \mathrm{d} x\right]^{1 / s^{\prime}}
\end{aligned}
$$


where $s^{\prime}=s /(s-1)$. If $s^{\prime}\left(\pi-\delta \alpha_{0}\right)<\pi$ we may apply Theorem A, to find a constant $C>0$ such that

$$
\int_{\mathbb{R}} Q(x)\left(e^{\alpha_{0} \tilde{u}_{n}^{2}}-1\right) \mathrm{d} x \leq \alpha_{0}\left\|\tilde{u}_{n}\right\|_{L^{2}(\mathbb{R} ; Q)}^{2}+C\left\|\tilde{u}_{n}\right\|_{L^{2 s}(\mathbb{R} ; Q)}^{2} .
$$

Now using compactness result from Lemma 2.1 in the above inequality, we get

$$
\int_{\mathbb{R}} Q(x)\left(e^{\alpha_{0} \tilde{u}_{n}^{2}}-1\right) \mathrm{d} x \rightarrow 0, \quad \text { as } \quad n \rightarrow \infty .
$$

The second integral in (5.8) may be estimated as follows: Considering $\beta_{1}>$ $\alpha_{0}$, we may find $C=C\left(\beta_{1}\right)>0$, such that for any $t \geq 0$

$$
g(t) \leq C e^{\beta_{1} t^{2}} .
$$

Hence

$$
\log \left(\frac{g(t)}{\sqrt{\alpha_{0}}}\right) \leq \log \left(\frac{C}{\sqrt{\alpha_{0}}}\right)+\beta_{1} t^{2}
$$

Using condition $(H 2)$, there exists a positive constant $C$ independent of $n$ such that

where

$$
v_{n}(x) \geq C \text { for all } x \in B_{n}
$$

$$
B_{n}:=\left\{x \in \mathbb{R}: g\left(v_{n}\right)(x) \geq \sqrt{\alpha_{0}} e^{1 / 4}\right\} .
$$

Thus using (5.10)-(5.11) we get

$$
\begin{aligned}
& \int_{B_{n}} Q(x) \frac{g\left(v_{n}\right)}{\sqrt{\alpha_{0}}}\left[\log \left(\frac{g\left(v_{n}\right)}{\sqrt{\alpha}_{0}}\right)\right]^{1 / 2} \mathrm{~d} x \\
& \leq \frac{1}{\sqrt{\alpha_{0}}} \sqrt{\log \left(\frac{C}{\sqrt{\alpha_{0}}}\right)} \int_{B_{n}} Q(x) g\left(v_{n}\right) \mathrm{d} x+\frac{\sqrt{\beta_{1}}}{\sqrt{\alpha_{0}}} \int_{\mathbb{R}} Q(x) g\left(v_{n}\right) v_{n} \mathrm{~d} x .
\end{aligned}
$$

We shall prove that

$$
\lim _{n \rightarrow \infty} \int_{B_{n}} Q(x) g\left(v_{n}\right) \mathrm{d} x=0 .
$$

Indeed, given $M>1$, we consider $A_{M}^{n}:=\left\{x \in \mathbb{R}: v_{n}(x) \geq M\right\}$. By $(H 1)$ and (H2), there exists $C>0$, independent of $n \in \mathbb{N}$, such that $g\left(v_{n}(x)\right) v_{n}(x) \leq$ $C v_{n}^{2}(x)$, for every $x \in B_{n} \backslash A_{M}^{n}$. Consequently, by (5.4),

$$
\begin{aligned}
& \int_{B_{n}} Q(x) g\left(v_{n}\right) \mathrm{d} x=\int_{B_{n} \backslash A_{M}^{n}} Q(x) g\left(v_{n}\right) \mathrm{d} x+\int_{B_{n} \cap A_{M}^{n}} Q(x) g\left(v_{n}\right) \mathrm{d} x \\
& \leq \int_{\left\{B_{n} \backslash A_{M}^{n}\right\} \cap B_{r}^{c}(0)} Q(x) g\left(v_{n}\right) \mathrm{d} x+\int_{\left\{B_{n} \backslash A_{M}^{n}\right\} \cap B_{r}(0)} Q(x) g\left(v_{n}\right) \mathrm{d} x \\
& +\frac{1}{M} \int_{\mathbb{R}} Q(x) g\left(v_{n}\right) v_{n} \mathrm{~d} x .
\end{aligned}
$$

Let $\epsilon>0$. For $L$ large enough and using (5.11), (H2), the Hölder inequality together with $Q(x)<\epsilon$ for $|x|>L$, we have for some constant $\tilde{C}>0$

$$
\int_{\left\{B_{n} \backslash A_{M}^{n}\right\} \cap B_{L}^{c}(0)} Q(x) g\left(v_{n}\right) \mathrm{d} x \leq \epsilon C \int_{\left\{B_{n} \backslash A_{M}^{n}\right\} \cap B_{L}^{c}(0)}\left|v_{n}\right|^{2} \mathrm{~d} x \leq \tilde{C} \epsilon .
$$


From compact embeddings, we have also for a fixed $L>0$

$$
\int_{\left\{B_{n} \backslash A_{M}^{n}\right\} \cap B_{L}(0)} Q(x) g\left(v_{n}\right) \mathrm{d} x=o_{n}(1) .
$$

Using above estimates and noting that $M>1$ may be chosen properly large, the above inequality implies that (5.13) must hold.

From (5.12) and (5.13) we obtain

$$
\int_{B_{n}} Q(x) \frac{g\left(v_{n}\right)}{\sqrt{\alpha_{0}}}\left[\log \left(\frac{g\left(v_{n}\right)}{\sqrt{\alpha}_{0}}\right)\right]^{1 / 2} \mathrm{~d} x \leq \sqrt{\frac{\beta_{1}}{\alpha_{0}}} \int_{\mathbb{R}} Q(x) g\left(v_{n}\right) v_{n} \mathrm{~d} x+\mathrm{o}_{n}(1) .
$$

Next, we use $(H 1)-(H 2)$ and Lemma 2.1 to obtain

$$
\frac{1}{2} \int_{\left\{x \in \mathbb{R}: g\left(v_{n}\right)(x) \leq \sqrt{\alpha_{0}} e^{1 / 4}\right\}} Q(x) \frac{\left(g\left(v_{n}\right)\right)^{2}}{\alpha_{0}} \mathrm{~d} x \leq \frac{C}{2 \alpha_{0}} \int_{\mathbb{R}} Q(x) v_{n}^{2} \mathrm{~d} x=o_{n}(1) .
$$

Again using Lemma 2.1, (5.9), (5.14), (5.15), we may write

$$
\left(\frac{\pi}{\alpha_{0}}-\delta\right)^{1 / 2}\left\|u_{n}\right\|_{1 / 2} \leq \mathrm{o}_{n}(1)+\sqrt{\frac{\beta_{1}}{\alpha_{0}}} \int_{\mathbb{R}} Q(x) g\left(v_{n}\right) v_{n} \mathrm{~d} x .
$$

Repeating the same argument for $v_{n}$, we also obtain

$$
\left(\frac{\pi}{\alpha_{0}}-\delta\right)^{1 / 2}\left\|v_{n}\right\|_{1 / 2} \leq \mathrm{o}_{n}(1)+\sqrt{\frac{\beta_{1}}{\alpha_{0}}} \int_{\mathbb{R}} P(x) f\left(u_{n}\right) u_{n} \mathrm{~d} x .
$$

Choosing $\beta_{1} \in\left(\alpha_{0}, \beta\right)$, the estimate (5.7) is a direct consequence of (5.6), (5.16), (5.17). The claim is proved.

Using (5.7) we can choose $\beta$ close enough to $\alpha_{0}$ and $\varepsilon>0$ such that $\left(\alpha_{0}+\varepsilon\right) \max \left\{\left\|u_{n}\right\|_{1 / 2}^{2},\left\|v_{n}\right\|_{1 / 2}^{2}\right\}<\pi$. Now using $(H 1)-(H 2)$ together with the exponential critical growth of $f$ and Theorem A we obtain

$$
\begin{aligned}
& \int_{\mathbb{R}} P(x) f\left(u_{n}\right) u_{n} \mathrm{~d} x \leq \varepsilon \int_{\mathbb{R}} P(x)\left|u_{n}\right|^{2} \mathrm{~d} x+C_{\varepsilon} \int_{\mathbb{R}} P(x) u_{n}\left(e^{\left(\alpha_{0}+\varepsilon\right) u_{n}{ }^{2}}-1\right) \mathrm{d} x \\
& \leq \epsilon\left\|u_{n}\right\|_{L^{2}(\mathbb{R} ; P)}^{2}+C_{\varepsilon}\left\|u_{n}\right\|_{L^{s}(\mathbb{R} ; P)}\left(\int_{\mathbb{R}} P(x)\left(e^{s^{\prime}\left(\alpha_{0}+\varepsilon\right) u_{n}{ }^{2}}-1\right) \mathrm{d} x\right)^{1 / s^{\prime}}
\end{aligned}
$$

where $1 / s+1 / s^{\prime}=1$. Now on choosing $s>2$ with $s^{\prime}$ sufficiently close to 1 such that $\left(\alpha_{0}+\varepsilon\right) \max \left\{\left\|u_{n}\right\|_{1 / 2}^{2},\left\|v_{n}\right\|_{1 / 2}^{2}\right\} s^{\prime}<\pi$, we can do the similar calculations as before to control the integral in the second term of the above inequality. Hence using compactness from Lemma 2.1, we obtain

$$
\int_{\mathbb{R}} P(x) f\left(u_{n}\right) u_{n} \mathrm{~d} x \rightarrow 0 .
$$

Analogously,

$$
\int_{\mathbb{R}} Q(x) g\left(v_{n}\right) v_{n} \mathrm{~d} x \rightarrow 0 .
$$

Finally, using (5.18), (5.19) and (5.2) we obtain

$$
\int_{\mathbb{R}}\left((-\Delta)^{\frac{1}{4}} u_{n}(-\Delta)^{\frac{1}{4}} v_{n}+u_{n} v_{n}\right) \mathrm{d} x \rightarrow 0
$$


which together with (5.5) gives a contradiction with (5.1). The proof of the theorem is complete.

\section{Acknowledgement}

Research was supported in part by INCTmat/MCT/Brazil, CNPq and CAPES/Brazil.

\section{Appendix: Validation of Remark 1.2}

In this appendix, we show the claim that under the assumptions $(H 2)$ and $(H 5), f$ (respectively $g$ ) belongs to the class $\mathbb{C}_{\mathrm{P}}$ (respectively $\mathbb{C}_{\mathrm{Q}}$ ) and that if $f(t)=O\left(t^{2}\right)$ then $u_{n} \rightarrow 0$ and $\left\{\int_{\mathbb{R}} P(x) f\left(u_{n}\right) u_{n} \mathrm{~d} x\right\}$ bounded imply that $\int_{\mathbb{R}} P(x) f\left(u_{n}\right) \mathrm{d} x \rightarrow 0$.

Proof. Let $\left\{u_{n}\right\} \subset H^{1 / 2,2}(\mathbb{R})$ be a sequence such that

$$
u_{n} \rightarrow 0, \text { and } \int_{\mathbb{R}} P(x) f\left(u_{n}\right) u_{n} \mathrm{~d} x<C .
$$

For any given $\epsilon>0$, using $(H 2)$ and $(H 5)$, there exists $c_{o}=c_{o}(\epsilon)>0$ sufficiently small and $M>0$ sufficiently large such that

$$
F(t) \leq \epsilon|t|^{2} \quad \text { for } \quad|t|<c_{o} \quad \text { and } \quad F(t) \leq \epsilon f(t) t \quad \text { for } t>M .
$$

Hence from (5.20), we have

$$
\begin{aligned}
\int_{\mathbb{R}} P(x) F\left(u_{n}\right) \mathrm{d} x \leq & \epsilon \int_{\left\{u_{n} \leq c_{o}\right\}} P(x)\left|u_{n}\right|^{2} \mathrm{~d} x+\int_{\left\{c_{o} \leq u_{n} \leq M\right\}} P(x) F\left(u_{n}\right) \mathrm{d} x \\
& +\epsilon \int_{\left\{u_{n} \geq M\right\}} P(x) f\left(u_{n}\right) u_{n} \mathrm{~d} x \\
\leq & \epsilon C+\int_{\left\{c_{o} \leq u_{n} \leq M\right\}} P(x) F\left(u_{n}\right) \mathrm{d} x .
\end{aligned}
$$

Further for $L=L(\epsilon)>0$ large enough such that $P(x)<\epsilon$ for $x \in B_{L}^{c}(0)$, we have

$$
\int_{\left\{c_{o} \leq u_{n} \leq M\right\} \cap B_{L}^{c}(0)} P(x) F\left(u_{n}\right) \mathrm{d} x \leq \epsilon C,
$$

where $C$ is independent of $n$. Indeed,

$$
c_{o}^{2}\left|\left\{c_{o} \leq u_{n} \leq M\right\}\right| \leq \int_{\left\{c_{o} \leq u_{n} \leq M\right\}}\left|u_{n}\right|^{2} \mathrm{~d} x \leq \int_{\mathbb{R}}\left|u_{n}\right|^{2} \mathrm{~d} x \leq C .
$$

Now by Lebesgue theorem, for a such fixed $L>0$, we have also

$$
\int_{\left\{c_{o} \leq u_{n} \leq M\right\} \cap[-L, L]} P(x) F\left(u_{n}\right) \mathrm{d} x \rightarrow 0 .
$$


22 Joao Marcos do Ó, Jacques Giacomoni and Pawan Kumar Mishra

Gathering (5.21)-(5.23), we get

$$
\int_{\mathbb{R}} P(x) F\left(u_{n}\right) \mathrm{d} x \rightarrow 0 .
$$

It finishes the proof of the first part of the claim. Next, we show the second statement of the claim. From $f(t)=O\left(t^{2}\right)$ and for $c_{o}, M>0$ respectively small and large enough, we have

$$
\begin{aligned}
\int_{\mathbb{R}} P(x) f\left(u_{n}\right) \mathrm{d} x= & \int_{\left\{u_{n}<c_{o}\right\}} P(x) f\left(u_{n}\right) \mathrm{d} x+\int_{\left\{c_{o} \leq u_{n} \leq M\right\}} P(x) f\left(u_{n}\right) \mathrm{d} x \\
& +1 / M \int_{\left\{u_{n}>M\right\}} P(x) f\left(u_{n}\right) u_{n} \mathrm{~d} x \\
\leq & C \int_{\mathbb{R}} P(x) u_{n}^{2} \mathrm{~d} x+\int_{\left\{c_{o} \leq u_{n} \leq M\right\}} P(x) f\left(u_{n}\right) \mathrm{d} x+C / M .
\end{aligned}
$$

Using Lemma 2.1 and estimating the second integral in the above inequality in a similar way as in (5.22) and (5.23), we get the required result.

Remark 5.1. Let the function $P$ be defined as $P(x)=\frac{1}{(|x|+1)^{\epsilon}}$, for $\epsilon>0$ sufficiently small. Consider the sequence $\left\{u_{n}\right\} \subset H^{1 / 2,2}(\mathbb{R})$ such that

$$
u_{n}(x)= \begin{cases}\frac{1}{n^{\alpha}}, & \text { for } x \in[n, 2 n], \\ \frac{(x-(n-1))}{n^{\alpha}}, & \text { for } x \in[n-1, n], \\ \frac{((2 n+1)-x)}{n^{\alpha}}, & \text { for } x \in[2 n, 2 n+1], \\ 0 & \text { elsewhere }\end{cases}
$$

with $\alpha \in[1 / 2,1)$. Then, by straighforward calculations, we can prove $\left\{u_{n}\right\}$ is bounded in $H^{1 / 2,2}(\mathbb{R})$. Furthermore, as $n \rightarrow \infty$

$$
\int_{\mathbb{R}} P(x) u_{n}^{q} \mathrm{~d} x \rightarrow 0
$$

if and only if $q$ satisfies $\alpha q+\epsilon>1$. Therefore if $f$ is of $O\left(t^{1+\ell}\right)$ near 0 with $0<\ell \leq \frac{1-\alpha-\epsilon}{\alpha}$, we easily get that $u_{n} \rightarrow 0$ weakly in $H^{1 / 2,2}(\mathbb{R})$ and

$$
\int_{\mathbb{R}} P(x) f\left(u_{n}\right) u_{n} \mathrm{~d} x \rightarrow 0
$$

as $n \rightarrow \infty$. However, $\int_{\mathbb{R}} P(x) f\left(u_{n}\right) \mathrm{d} x \rightarrow 0$ is not verified.

\section{References}

[1] W. Abdelhedi and H. Chtioui. On a Nirenberg-type problem involving the square root of the Laplacian. Journal of Functional Analysis, 265: 2937-2955, 2013.

[2] V. Ambrosio, G. M. Bisci, and D. Repovš. Nonlinear equations involving the square root of the Laplacian. Discrete $\&$ Continuous Dynamical Systems - S, 12 (2) : 151-170, 2019.

[3] V. Benci and P. H. Rabinowitz. Critical point theorems for indefinite functionals. Invent. Math., 52(3):241-273, 1979. 
[4] D. Bonheure, E. M. dos Santos, and H. Tavares. Hamiltonian elliptic systems: a guide to variational frameworks. Port. Math., 71(3-4):301-395, 2014.

[5] X. Cabré and J. Tan. Positive solutions of nonlinear problems involving the square root of the Laplacian. Advances in Mathematics, 224: 2052-2093, 2010.

[6] P. Clément, D. G. de Figueiredo, and E. Mitidieri. Positive solutions of semilinear elliptic systems. Comm. Partial Differential Equations, 17(5-6):923-940, 1992.

[7] D. G. de Figueiredo, J. M. do Ó, and B. Ruf. Critical and subcritical elliptic systems in dimension two. Indiana Univ. Math. J., 53(4):1037-1054, 2004.

[8] D. G. de Figueiredo, J. M. do Ó, and B. Ruf. An Orlicz-space approach to superlinear elliptic systems. J. Funct. Anal., 224(2):471-496, 2005.

[9] D. G. de Figueiredo, J. M. do Ó, and J. Zhang. Ground state solutions of hamiltonian elliptic systems in dimension two. preprint, 2017.

[10] D. G. de Figueiredo and P. L. Felmer. On superquadratic elliptic systems. Trans. Amer. Math. Soc., 343(1):99-116, 1994.

[11] D. G. de Figueiredo and J. Yang. Decay, symmetry and existence of solutions of semilinear elliptic systems. Nonlinear Anal., 33(3):211-234, 1998.

[12] D. G. de Figueiredo, J. M. do Ó, and B. Ruf. Critical and subcritical elliptic systems in dimension two. Indiana Univ. Math. J., 53(3):1037-1054, 2004.

[13] M. de Souza and J. M. do Ó. Hamiltonian elliptic systems in $\mathbb{R}^{2}$ with subcritical and critical exponential growth. Ann. Mat. Pura Appl. (4), 195(3):935-956, 2016.

[14] E. Di Nezza, G. Palatucci, and E. Valdinoci. Hitchhiker's guide to the fractional Sobolev spaces. Bull. Sci. Math., 136(5):521-573, 2012.

[15] J. M. do Ó, O. H. Miyagaki, and M. Squassina. Nonautonomous fractional problems with exponential growth. NoDEA Nonlinear Differential Equations Appl., 22(5):1395-1410, 2015.

[16] J. Giacomoni, P. K. Mishra, and K. Sreenadh. Critical growth problems for 1/2-Laplacian in $\mathbb{R}$. Differential Equations $\mathcal{E}$ Applications, 8(3):295-317, 2016.

[17] J. Giacomoni, P. K. Mishra, and K. Sreenadh. Fractional elliptic equations with critical exponential nonlinearity. Adv. Nonlinear Anal., 5(1):57-74, 2016.

[18] J. Hulshof and R. van der Vorst. Differential systems with strongly indefinite variational structure. J. Funct. Anal., 114(1):32-58, 1993.

[19] A. Iannizzotto and M. Squassina. 1/2-Laplacian problems with exponential nonlinearity. J. Math. Anal. Appl., 414: 372-385, 2014.

[20] W. Kryszewski and A. Szulkin. An infinite-dimensional Morse theory with applications. Trans. Amer. Math. Soc., 349(8):3181-3234, 1997.

[21] N. Lam and G. Lu. Elliptic equations and systems with subcritical and critical exponential growth without the Ambrosetti-Rabinowitz condition. J. Geom. Anal., 24(1):118-143, 2014.

[22] G. Li and J. Yang. Asymptotically linear elliptic systems. Comm. Partial Differential Equations, 29(5-6):925-954, 2004.

[23] L. Martinazzi. Fractional Adams-Moser-Trudinger type inequalities. Nonlinear Anal., 127:263-278, 2015.

[24] T. Ozawa. On critical cases of Sobolev's inequalities. J. Funct. Anal., 127(2):259-269, 1995. 
[25] P. H. Rabinowitz. Some global results for nonlinear eigenvalue problems. J. Functional Analysis, 7:487-513, 1971.

[26] P. H. Rabinowitz. Periodic solutions of Hamiltonian systems. Comm. Pure Appl. Math., 31(2):157-184, 1978.

[27] P. H. Rabinowitz. Minimax methods in critical point theory with applications to differential equations, volume 65 of CBMS Regional Conference Series in Mathematics. Published for the Conference Board of the Mathematical Sciences, Washington, DC; by the American Mathematical Society, Providence, RI, 1986.

[28] E. A. B. de Silva. Linking theorems and applications to semilinear elliptic problems at resonance. Nonlinear Anal., 16(5):455-477, 1991.

[29] B. Sirakov. On the existence of solutions of Hamiltonian elliptic systems in $\mathbb{R}^{N}$. Adv. Differential Equations, 5(10-12):1445-1464, 2000.

[30] B. Sirakov and S. H. M. Soares. Soliton solutions to systems of coupled Schrödinger equations of Hamiltonian type. Trans. Amer. Math. Soc., 362(11):5729-5744, 2010.

[31] F. Takahasi. Critical and subcritical fractional Trudinger-Mosertype inequalities on $\mathbb{R}$. Adv. Nonlinear Anal., to appear DOI: https://doi.org/10.1515/anona-2017-0116

[32] J. Tan. The Brezis-Nirenberg type problem involving the square root of the Laplacian. Calc. Var. Partial Differential Equations, 36(1-2): 21-41, 2011.

[33] X. Yu. The Nehari manifold for elliptic equation involving the square root of the Laplacian. J. Differential Equations, 252 :1283-1308, 2012.

João Marcos do Ó

e-mail: jmbo@pq.cnpq.br

(Joao Marcos do Ó) Department of Mathematics, Brasília University, 70910-900, Brasília, DF, Brazil

Jacques Giacomoni

e-mail: jacques.giacomoni@univ-pau.fr

(Jacques Giacomoni) LMAP (UMR E2S-UPPA CNRS 5142), Bat. IPRA, Avenue de l'Université, F-64013 Pau, France

Pawan Kumar Mishra

e-mail: pawanmishra@mat.ufpb.br

(Pawan Kumar Mishra) Department of Mathematics, Federal University of Paraíba, 58051-900, João Pessoa-PB, Brazil 